\title{
Fluid-Structure Interaction Analysis on Cantilever Beams for Micro- Energy Harvesting of Cross-flow Turbine
}

\author{
Yeong Wan Je ${ }^{1}$ and Youn-Jea Kim² \\ ${ }^{1}$ Graduate School of Mechanical Engineering, Sungkyunkwan University \\ Suwon 16419, Republic of Korea \\ ywje@skku.edu \\ ${ }^{2}$ School of Mechanical Engineering, Sungkyunkwan University \\ Suwon 16419, Republic of Korea \\ yjkim@skku.edu
}

\section{Extended Abstract}

The harvested electrical energy is used in a variety of electronic equipment such as remote sensors, automobiles, medical or military equipment, etc. As the energy demand increases, the need for an efficient energy harvesting system increases and the relevant researches are actively carried out [1-5]. Cross-flow turbine is a water impulse turbine with relatively low efficiency, but it can be adjusted at various flow rates and is easy to maintain. As the working fluid passes through the impeller of the cross-flow hydraulic turbine and forms a vortex field at downstream, the induced vortex flow can be used for converting the kinetic energy inherent in vibrations to electricity using energy harvesters such as cantilevers, membranes or other structures. In this study, the cantilever beams were located at the downstream of cross-flow hydraulic turbine for microenergy harvesting. Numerical analysis was conducted using the commercial code, ANSYS CFX 18.1 with the k- $\omega$ based shear stress transport (SST) turbulence model. The effect of distance between cantilever beams on stress and strain was evaluated using 2-way fluid-structure interaction (FSI) analysis. As a result, the maximum von-Mises stress of the cantilever beam was calculated as $163.5 \mathrm{MPa}$, and the maximum deformation was calculated as $2.29 \mathrm{~mm}$. In addition, the results were graphically depicted with various geometrical and flow conditions.

\section{References}

[1] D. Shen, J. Park, J. Ajitsaria, S. Choe, H. C Wikle III and D. Kim, "The design, fabrication and evaluation of a MEMS PZT cantilever with an integrated Si proof mass for vibration energy harvesting," J. Micromech. Microeng. vol. 18, no. 5, pp. 1-7, 2008.

[2] D. Wang, C. Chiu and H. Pham, "Electromagnetic energy harvesting from vibrations induced by Kármán vortex street," J. Mechatronics, vol. 22, pp. 746-756, 2012.

[3] P. Rani and D. Chhabra, "Piezoelectric Energy Harvesting from Fluid Flow Dynamism using PVDF," J. R\&D in Engineering, Science and Management, vol. 4, pp. 22-36.

[4] P. Wang, X. Dai, D. Fang and X. Zhao, "Design, fabrication and performance of a new vibration-based electromagnetic micro power generator," J. Microelectronics, vol. 38, pp. 1175-1180, 2007.

[5] H. Fang, J. Liu, Z. Xu, L. Dong, L. Wang, D. Chen, B. Cai and Y. Liu, "Fabrication and performance of MEMS-based piezoelectric power generator for vibration energy harvesting," J. Microelectronics, vol. 38, pp. 1280-1284, 2007. 